\title{
ON THE DIRICHLET PROBLEM FOR LINEAR DIFFERENTIAL EQUATIONS
}

\section{J. L. ERICKSEN}

In nonlinear elasticity, it is of interest to know for what real constants $\lambda$ and $\mu$ the system of "linear elasticity equations"

$$
\sum_{k=1}^{n}\left[(\lambda+\mu) \frac{\partial^{2} u_{k}}{\partial x_{k} \partial x_{i}}+\mu \frac{\partial^{2} u_{i}}{\partial x_{k}^{2}}\right]=0, \quad(i=1, \cdots, n>1)
$$

admits a unique solution to the Dirichlet problem for sufficiently smooth regions and boundary data. This question also appears to be of some interest in the general theory of partial differential equations.

The system (1) is elliptic if and only if $(\lambda+2 \mu) \mu \neq 0$, strongly elliptic if and only if $(\lambda+2 \mu) \mu>0$. When it is strongly elliptic, existence and uniqueness of solutions to the Dirichlet problem for a rather general class of regions and boundary data can be inferred from results of Browder [1] and Morrey [2]. It is noted in [3] that uniqueness fails when $(\lambda+2 \mu) \mu=0$. For the elliptic systems obtained from (1) by setting $n=2, \lambda=-3 \mu \neq 0$ and replacing $u_{2}$ by $-u_{2}$ or $u_{2}$ by $2^{1 / 2} u_{2}$, Biçadze [4] has shown that uniqueness fails. To settle the question of uniqueness, it thus remains to dispose of the case $(\lambda+2 \mu) \mu<0$, which we do in the following

Theorem. When $(\lambda+2 \mu) \mu<0$, the system (1) will admit nonzero solutions which vanish on the boundary of some region $R$ of given diameter and are analytic in the closure of $R$.

Proof. It is easily verified by direct substitution in (1) that

$$
u_{1}=(n-1) \mu x_{1}^{2}-(\lambda+2 \mu) \sum_{k=2}^{n} x_{k}^{2}-\epsilon \mu, \quad u_{2}=\cdots=u_{n}=0,
$$

where $\epsilon$ is any positive constant, is a solution of (1) which is analytic everywhere. When $(\lambda+2 \mu) \mu<0$, the surface $u_{1}=0$ is an ellipsoid and one can choose $\epsilon$ so that the region $R$ interior to it has any prescribed diameter.

\section{REFERENCES}

1. F. E. Browder, Strongly elliptic systems of differential equations, Annals of Mathematics Studies, vol. 33, 1954, pp. 15-51.

2. C. B. Morrey, Jr., Second order elliptic systems of differential equations, Annals of Mathematics Studies, vol. 33, 1954, pp. 101-159.

Received by the editors August 23, 1956. 
3. J. L. Ericksen and R. A. Toupin, Implications of Hadamard's condition for elastic stability with respect to uniqueness theorems, Canadian Journal of Mathematics vol. 8 (1956) pp. 432-436.

4. A. V. Biçadze, On the uniqueness of solutions of the Dirichlet problem for elliptic partial differential equations, Uspekhi Matematičeskih Nauk (N.S.) vol. 3 (1948) pp. 211-212.

Naval Research Laboratory, Washington, D. C.

\section{ON THE IDENTITIES OF CERTAIN ALGEBRAS}

\section{ADIL YAQUB}

Introduction. One basic problem in the study of finite algebras is concerned with the existence of a finite basis for the identities of the algebra, i.e., a finite set of identities from which all the identities of the algebra are logical consequences. That the identities of a finite algebra need not have a finite basis (in the above sense) has already been observed by Lyndon [3]. This leads naturally to the following question: are there certain classes of finite algebras the identities of which possess a finite basis? We shall answer this question in the affirmative. Indeed, the main result of this paper is the following

TheOREM. A functionally strictly complete algebra which contains more than one element has a finite basis for its identities.

1. Preliminary concepts. In this section, we shall review some basic concepts and definitions, all of which are to be found in Foster $[1 ; 2]$.

Let $\mathfrak{A}=(A, \rho, \cdots)$ be a universal algebra with primitive operations $\rho, \cdots$. Let $A=\{\cdots, x, \cdots\}$.

A (k-ary) 2-function $f\left(x_{1}, \cdots, x_{k}\right)$ is a composition, via the primitive operations, of indeterminate symbols $x_{1}, \cdots, x_{k}$ over the set $A$ together with a (possibly empty) set of $\operatorname{constants}^{1}$ ( $=$ fixed $\in A$ ).

An $\mathfrak{A}$-function is called strict if it involves no constants.

In an obvious way each $\mathfrak{A}$-function $f\left(x_{1}, \cdots, x_{k}\right)$ represents (or has associated with it) a mapping of the set $A^{k}$ into $A$, where of course different $\mathfrak{A}$-functions need not represent different such mappings. If $\mathfrak{A}$-functions $f(x, \cdots)$ and $g(x, \cdots)$ represent the same mapping we

Presented to the Society, April 14, 1956; received by the editors February 10, 1956 and, in revised form, August 25, 1956.

1 In more uniform terminology a constant may also be defined as a " 0 -ary" function. 